Sociologie et sociétés

\title{
L'hypothèse des délais, Sociologie ou artifice statistique? " Éducation, qualification professionnelle et carrière au Québec »
}

\section{Commentary The Hypothesis of Time-Lag - Sociology or Statistical Artifice}

François BÉLAND

Volume 16, numéro 2, octobre 1984

Sociétés et vieillissement

URI : https://id.erudit.org/iderudit/001033ar

DOI : https://doi.org/10.7202/001033ar

Aller au sommaire du numéro

Éditeur(s)

Les Presses de l'Université de Montréal

ISSN

0038-030X (imprimé)

1492-1375 (numérique)

Découvrir la revue

Citer cette note

BÉLAND, F. (1984). L'hypothèse des délais, Sociologie ou artifice statistique?

"Éducation, qualification professionnelle et carrière au Québec ". Sociologie et

sociétés, 16(2), 129-135. https://doi.org/10.7202/001033ar d'utilisation que vous pouvez consulter en ligne. 


\section{Débats*}

* Le lecteur trouvera plus ici un commentaire critique portant sur un texte publié dans un de nos précédents numéros. C'est là une première pour Sociologie et sociétés qui jusqu'ici ne publiait que des articles reliés au thème de chaque numéro. Nous espérons toutefois avoir l'occasion de publier ce genre de texte qui, par définition, devrait être court et concis. Autant que possible ces notes critiques seront accompagnées d'une réponse des auteurs faisant l'objet de ces commentaires.

ROBERT SÉVIGNY

\section{NOTE CRITIQUE}

\section{L'HYPOTHÈSE DES DÉLAIS, SOCIOLOGIE OU ARTIFICE STATISTIQUE*}

«Éducation, qualification professionnelle et carrière au Québec» (Jean Renaud, Paul Bernard et Monique Berthiaume, Sociologie et sociétés, vol. XII, $\mathbf{n}^{\circ} 1$, avril 1980).

\section{François Béland}

Quelles sont les composantes de la carrière professionnelle et comment s'articulent-elles dans l'histoire des occupations d'une cohorte? Renaud, Bernard et Berthiaume ${ }^{\prime}$ (1980) veulent faire eclater les notions «fourre-tout» qui ont servi à étudier cette question. Le statut socioéconomique (SSE) mesuré par des indices dits de prestige (Blau et Duncan, 1967; Blishen et McRoberts, 1976) a servi d'indicateur universel de la notion de carrière. Le SSE peut être critiqué d'au moins deux points de vue différents: d'une part, il n'est pas assez spécifique et d'autre part, il occulte la multi-dimensionnalité de la notion de carrière, décourageant les additions de composantes fondarnentales dans l'étude empirique de la distribution des emplois.

Le but du travail de RBB est de «tirer au clair les rapports entre éducation, qualification professionnelle et carrière» (25), toutes des dimensions de la carrière professionnelle. En une première partie, ils étudient la distribution des qualifications selon les secteurs de l'activité économique (31-36). Dans une deuxième partie, ils emploient la méthode connue des cheminements de causalité (Duncan 1975) pour étudier la part respective des différentes dimensions scolaires et de la qualification professionnelle spécifique dans la détermination de la carrière. Cette deuxième partie retiendra notre attention.

Dans une certaine mesure, la carrière apparaît définie comme un réseau de cheminement de causalité entre les quatre types de variables utilisées par RBB: les variables de scolarité, la variable de qualification professionnelle, la variable de statut socio-économique ou d'occupation et la variable de revenu. Le statut d'occupation est retenu comme une variable indiquant «assez généralement les propriétés hiérarchiques des occupations» (40).

En introduisant la qualification professionnelle cependant, RBB cherche à résoudre trois problèmes: 1) «mieux comprendre ce que représente en réalité la variable fourre-tout qu'est le statut, en montrant qu'il dépend causalement, probablement dans une large mesure, de la qualification» $(40), 2)$ rendre compte de l'inertie des status et des revenus et 3) vérifier si la qualification ajoute à «la précision de nos connaissances sur le déroulement de la carrière» (41).

Mais les rapports empiriques découverts par RBB complexifient singulièrement les rapports que l'on voulait mieux comprendre et préciser. En effet, RBB observent une relation négative

* La recherche ASOPE a assuré les services informatiques nécessaires à la rédaction de cette note. Celle-ci a été rendue possible en partie grâce à la bourse de chercheur du Fonds de recherche en santé du Québec.

1. Ci-après udentıtié comme RBB. Les chiffres entre parenthèses réfèreront ici aux pages de leur article. 
entre la qualification professionnelle en début de carrière et le statut professionnel atteint à l'âge de 35 ans dans un sous-échantillon masculin de la main-d'ouvre québécoise.

L'interprétation de cette relation ne va pas sans problème. Après avoir rejeté la possibilité qu'elle soit l'effet de la multicollinéarité (46), plusieurs processus sont évoqués qui aboutissent à considérer une hypothèse susceptible de l'expliquer (47-48). Dans cette note critique, la vraisemblance de cette hypothèse sera réétudiée et la possibilité que la relation négative soit due à la multicollinéarité, réévaluée.

\section{L'HYPOTHÈSE}

La vraisemblance de l'hypothèse ne sera pas examinée sous l'angle d'une théorie du cheminement de carrière et du rôle de la qualification professionnelle opposée à celle de RBB. Plutôt, les variations des relations entre la qualification professionnelle et le statut socio-professionnel au début de la carrière et à 35 ans seront revues à la lumière de l'hypothèse de RBB ${ }^{2}$. Cette hypothèse est la suivante: il y a deux processus d'accès à un statut d'emploi quelconque à 35 ans: a) le processus que suivent la plupart des travailleurs, soit la transformation de la qualification initiale (PPS1) en occupation au début de la carrière (SEE1) et l'augmentation de la qualification à 35 ans (PPS35) qui se traduit par une augmentation du statut professionnel à cet âge (SSE35). On peut supposer que les corrélations entre ces variables sont ici toutes positives. Les moins qualifiés et ceux possédant un statut professionel bas en début de carrière obtiennent une occupation d'un rang moins élevé à 35 ans que les plus qualifiés et que ceux ayant un SSE élevé en début de carrière. b) Le deuxième processus concerne un sous-groupe de travailleurs qui, sans passer par les voies ordinaires de la mobilité sociale ascendante, augmentent leur statut professionnel à 35 ans (SSE35) en comblant des postes vacants (48). Ce processus s'observe surtout chez les moins qualifiés qui accèdent ainsi à des emplois supérieurs à ceux de leurs emplois en début de carrière.

Puisque la corrélation simple, calculée depuis les coefficients de cheminement de causalité de RBB, entre la qualification au premier emploi (PPS1) et le statut à 35 ans (SSE35) est positive, la projection dans un plan de la distribution conjointe observée de ces deux variables devrait ressembler à l'une des deux figures du graphique 1. Les courbes a et a' représentent les cheminements habituels: les personnes qui ont une qualification supérieure en début de carrière atteignent en général un SSE élevé à 35 ans. Les courbes $b$ et $b^{\prime}$ représentent deux façons possibles pour les travailleurs non qualifiés d'atteindre un SSE plus élevé à 35 ans que le SSE moyen des individus de même qualification d'origine. Dans le premier cas, (graphique la), les individus qui comblent des vacances réalisent un SSE moyen plus élevé que le SSE moyen atteint par des individus de même statut occupationnel d'origine. Mais cet effet diminue avec l'augmentation de la qualification de telle sorte que les gains de statut diminuent absolument avec une qualification de départ supérieure. Dans le deuxième cas, les individus qui comblent des vacances ont une augmentation de SSE plus élevée que l'augmentation moyenne des SSE atteints par le groupe entier des individus de même qualification d'origine, mais il n'y a ici que diminution relative de la moyenne des gains. Dans l'un et l'autre cas, la corrélation simple entre PPS1 et SSE35 sera positive. Clairement, il y aurait donc deux types de relation entre ces variables. La vérification de l'hypothèse de RBB exige l'observation de cette double spécification du rapport entre ces deux variables. On peut noter que dans l'un et l'autre cas, l'hypothèse implicite d'homoscédasticité en régression linéaire ne tiendra plus puisque la variation de SSE35 autour des valeurs faibles de PPS1 est plus importante que celle autour des valeurs élevées (les coefficients de régression multiple sont les estimateurs des paramètres des cheminements de causalité dans le cas des modèles récursifs (Carlos, 1970; Duncan, 1975)). Cependant la corrélation entre les qualifications professionnelles en début de carrière (PPS1) et les résidus de la prédiction du SSE35 par PPS35 pourra être presque nulle puisqu'un équilibre peut s'établir entre les déviations positives et négatives (graphique 2). Ce qui implique un coefficient de régression multiple très faible entre PPS1 et SSE35, dû en partie à la présence du groupe b selon l'hypothèse des délais de RBB. En effet, le coefficient de la régression simple de SSE35 sur PPS1 pourra être plus faible que celui de la régression simple de SSE35 sur PPS35. La variation

2. Les variables employées par RBB et leurs symboles respectifs sont: FG: formation générale; FP: formation professionnelle; PPS 1: qualification professionnelle à l'entrée sur le marché du travail; SSE1: statut professionnel à l'entrée; REV1: revenu à l'entrée; PPS35: qualification professionnelle à 35 ans; SSE35: statut professionnel à 35 ans; REV35: revenu à 35 ans. 
des SSE35 autour de la ligne de régression sera probablement plus grande dans le premier cas que dans le second. Aussi, la prédiction de SSE35 par PPS35 sera beaucoup plus adéquate et fortement positive. Cependant, la variation des résidus de cette prédiction autour de PPS35 sera plus prononcée pour les individus à faible qualification à 35 ans (graphique 3).

Mais ce n'est pas l'examen de la forme de la relation entre PPS1 et SSE35 qui a amené RBB à considérer l'hypothèse des délais, mais bien l'existence de la transformation de la corrélation positive entre PPS1 et SSE35 en effet causal négatif de PPS1 sur SSE35 lorsque les variables de formation, de statut professionnel en début de carrière et de qualification professionnelle à 35 ans sont introduites dans l'analyse.

Puisque l'hypothèse de la double spécification des rapports entre PPSI et SSE35 (graphique 1) traduit formellement l'idée de délais, est-ce que les relations entre les variables de RBB permettent de déduire un effet causal négatif entre PPS1 et SSE35 étant donné l'état théorique (graphiques 1 à 3) et observé des rapports entre variables stratégiques?

Graphique 1. Les relations entre PPS1 et SSE35
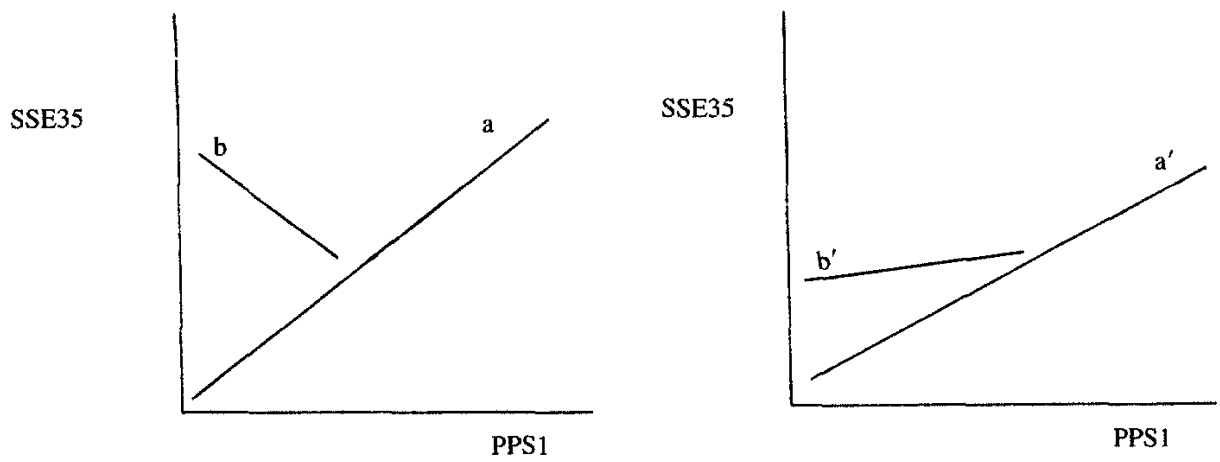

Graphique 1a

Graphique $1 b$

Graphique 2. Distribution des résidus de la prédiction de SSE35 par PPS ${ }^{1}$

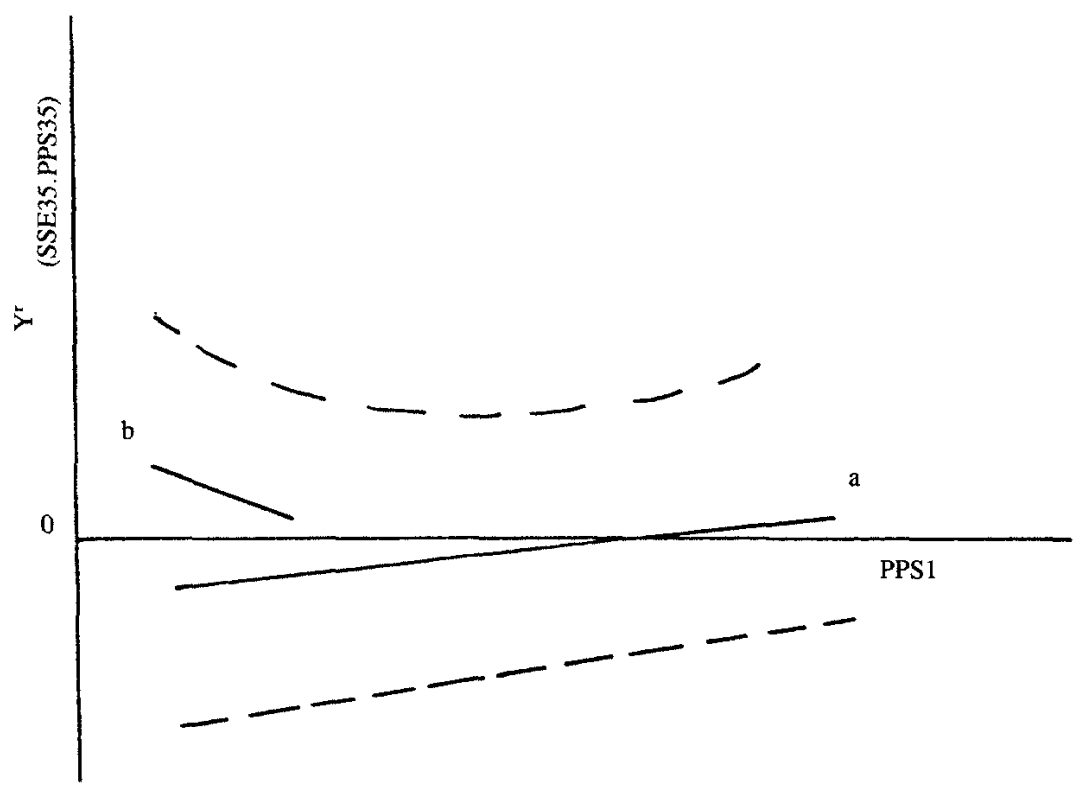

1 La zone ombragée indique la dispersion probable des cas.

a: processus habituel; b: processus des délais. 
Graphique 3. Distribution des résidus de la prédiction de SSE35 par PPS351

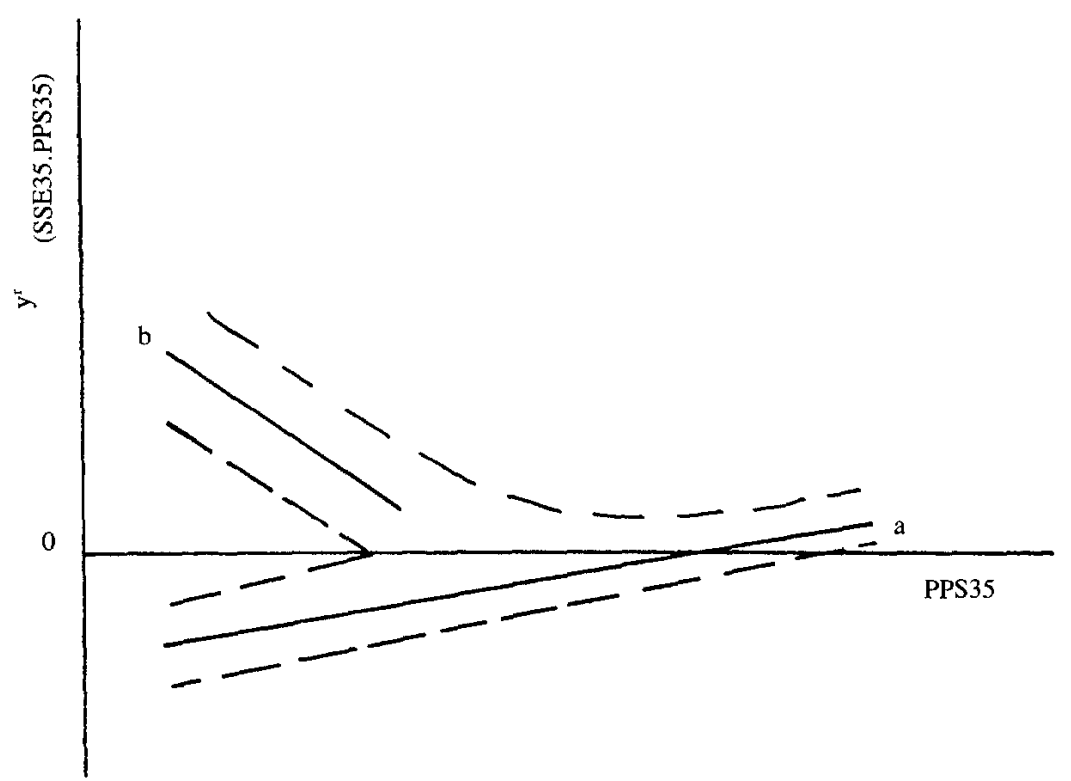

1 La zone ombragée indique la dispersion probable des cas. a: processus habituel; $b$ : processus des délais.

Un premier argument est de rigueur. RBB ont utilisé une mesure de la qualification professionnelle des emplois plutôt que des individus, attribuant le pointage de chaque occupation à ses détenteurs dans leur échantillon. La qualification professionnelle appartient donc à I'occupation plutôt qu'à l'individu (30-31). Les remarques qui suivent supposent plutôt que la qualification professionnelle est mesurée auprès de l'individu, tout comme la théorie de RBB qui suppose des mouvements différents de la qualification professionnelle et de l'occupation détenue. ce qui suit cherche à vérifier si l'hypothèse de RBB supporte l'effet négatif de PPS1 sur SSE35 au-delà des indicateurs et instruments de mesure, mais en supposant l'obtention d'une matrice de corrélations avec des données adéquates à notre propos.

La variable la plus intéressante pour étudier l'effet négatif est à notre avis la qualification à 35 ans. En effet, les travailleurs moins qualifiés qui profitent du délai (groupe b, graphiques 1-4) n'ont pas augmenté leur qualification à 35 ans. Ce sont des individus qui «voient leur qualification et leur statut évoluer de façon discordante» $(47 .$.$) ils ne passent pas «par les circuits usuels, c'est-$ à-dire l'enchaînement des préparations professionnelles spécifiques et celui des statuts» (48). Donc, la corrélation entre leur qualification en début de carrière et celle à 35 ans est négative (graphique la) ou faiblement positive (graphique $1 \mathrm{~b}$ ), tandis que la plupart des autres individus l'ont augmentée selon une tendance linéaire positive, mais peut-être à un taux différent selon la qualification et le statut de l'occupation d'origine. La relation bi-variée entre PPS35 et SSE35 est donc de même forme que celle entre PPS1 et SSE35. Des figures semblables à celle du graphique 1 peuvent être composées pour illustrer les relations théoriques possibles entre PPS35 et SSE35. Les résidus étant cependant plus correlés avec la variable prédictrice (graphique 3 ).

Lorsqu'une régression linéaire multiple est effectuée, les coefficients obtenus représentent en fait la relation qui existe entre les résidus de deux régressions multiples, soit 1) une régression d'une des variables indépendantes sur les autres et 2) une régression de la variable dépendante sur les autres variables indépendantes (Draper et Smith, 1966). Tel que, si X est une variable dépendante, $A$ une variable indépendante et $B$ un vecteur de variables indépendantes, la régression partielle de $X$ sur $A$ peut s'exprimer par une série d'équations:

(1) $\mathrm{A}=\mathrm{f}(\mathrm{B})+\mathrm{e}_{1}$

d'où

(2) $X=f(B)+e_{2}$

(3) $\beta(X . A) \mid B=f\left(e_{1}, e_{2}\right)$ 
quand l'équation 1 représente la première des deux régressions multiples, l'équation 2, la seconde et l'équation 3 montre que le coefficient de régression multiple de $\mathrm{X}$ sur $\mathrm{A}$ est une fonction des résidus des deux équations.

Il est aisé de remplacer les variables X, A et B par les variables SSE35, PPS1 et PPS35, tel que l'effet de PPS1 sur SSE35, étant donné PPS35 s'écrit:

(1) PPS1 $=\mathrm{f}\left(\right.$ PPS35) $+\mathrm{e}_{1}$

(2) SSE35 $=\mathrm{f}\left(\right.$ PPS35) $+\mathrm{e}_{2}$

et

(3) $\beta$ (S35.P1) $\mid$ P35 = f(e $\left.e_{1}, e_{2}\right)$

L'observation d'un coefficient négatif entre PPS1 et SSE35 étant donné la qualification à 35 ans (PPS35) peut en effet s'expliquer par l'effet double entre PPS1 et PPS35 d'une part, et PPS35 et SSE35 d'autre part. Pour simplifier l'exposé, seules les variables indépendantes PPS1 et PPS35 seront utilisées ici. L'utilisation de PPS1 dans la prédiction de SSE35 produit un coefficient de régression standardisé de .101 contre un coefficient de .692 entre PSS35 et SSE35; 1'introduction de SSE1 dans l'équation rend négatif ce premier coefficient, -.190 , et diminue à .561 le second. Il est manifeste que l'introduction d'autres variables ne fait qu'accentuer cette tendance des coefficients de régression partielle de SSE35 sur PPS1 et PPS35.

L'effet de PPS 1 sur SSE35 étant donné PPS35 symbolisé par $\beta_{(\mathbf{S 3 5 . P 1 ) | P 3 5}}$ peut donc s'exprimer comme la régression des résidus de la régression de SSE35 sur PPS35 et des résidus de la régression de PPS 1 sur PPS35. L'effet de PPS35 sur SSE35 étant donné PPS1 (symbolisé par $\beta_{(\mathrm{S} 35 . \mathrm{P} 35) \mid \mathrm{P} 1}$ peut se définir de la même manière. Il n'y a aucune raison de croire que la régression de PPS1 sur PPS35 ou de l'inverse soit biaisée de quelque manière, en conséquence seul le comportement théorique des résidus des régressions simples de SSE35 sur PPS1 d'une part, et de PPS35 de l'autre, sera examiné.

De façon générale, $\boldsymbol{\beta}_{(535 . \mathrm{P}) \mid \mathrm{P} 35}$ sera positif si les résidus de la régression de SSE35 sur PPS35 et ceux de la régression de PPS1 sur PPS35 vont dans le même sens, il sera négatif dans le cas contraire et nul s'il n'y a aucune relation entre eux. Le même raisonnement s'applique dans le cas de $\beta_{(\mathrm{S} 35 . \mathrm{P3} 5) \mid \mathrm{PI}}$.

La perte $\beta_{(\mathrm{S} 35 \mathrm{P} 1) \mid \mathrm{P35}}$ peut être illustrée de la même façon que la pente $\beta_{\mathrm{S} 35 . \mathrm{P} 1}$ au graphique $1 \mathrm{~b}$. Tandis que le coefficient $\beta_{\mathrm{S} 35 . \mathrm{P} 1}$ est empiriquement positif, l'estimation de $\beta_{(\mathrm{S35.P1)|P35}}$ tend à être nulle ou légèrement négative. Au graphique 4 , il apparaît que seule la présence du groupe b peut expliquer cette valeur négative. L'introduction de PPS35 dans le modèle réduit l'impact du groupe a dans l'équation. L'hypothèse des délais s'exprime donc formellement de façon relativement simple. Qui plus est, les résidus autour de la pente $\boldsymbol{\beta}_{(\mathrm{S} 35 . \mathrm{P}) \mid \mathrm{P} 35}$ ont une forme caractéristique: ils sont négatifs pour les valeurs faibles de $\mathrm{Y}^{\mathrm{r}}$ (SSE35.PPS35), nuls pour les valeurs moyennes de $\mathrm{Y}^{\mathrm{r}}$ (SSE35.PPS35) et positives pour les valeurs fortes de $\mathrm{Y}^{\mathrm{r}}$ (SSE35.PPS35). Remplacer cette dernière expression par le statut socioéconornique à 35 ans (SSE35) donnerait probablement une bonne approximation des biais des

Graphique 4. Pente de la régression multiple des SSE35 sur PPS1 et sa décomposition théorique entre les groupes a et b

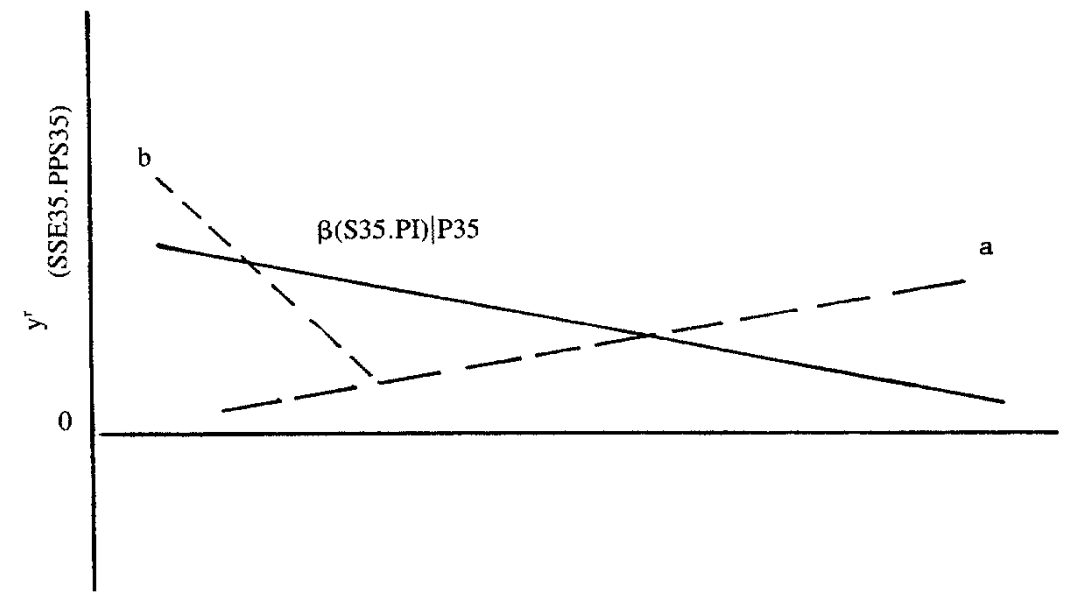


résidus. Dans ce cas, l'hypothèse des délais (i.e. de l'existence du groupe b) se vérifie dans la mesure où les erreurs de prédiction du statut à 35 ans (SSE35) selon le modèle de cheminement de causalité du schéma 1 de RBB, sont positivement liées à ce statut. Ce rapport aurait dû être vérifié par RBB.

L'hypothèse des délais peut donc produire une diminution sensible du rapport entre PPS1 et SSE35 lorsque PPS35 est introduit dans l'analyse. Cette diminution se convertirait en rapport négatif au fur et à mesure de l'introduction des autres variables du modèle. Cette relation négative n'est cependant qu'un signal qui exige une observation plus poussée de données, c'est-à-dire audelà d'une interprétation simplement plausible d'un coefficient négatif. Cette observation peut être menée à l'aide d'un modèle conforme à l'hypothèse d'une accession tardive à des occupations de statut moyen ou élevé par des travailleurs qui n'augmentent pas leur qualification professionnelle. Les individus appartenant à ce groupe peuvent être identifiés par leur position sur des graphiques observés équivalents aux graphiques théoriques 1 à 4 . Les frontières entre les groupes a et $b$ (voir les graphiques) peuvent être empiriquement plus ou moins difficiles à tracer, mais les tendances devraient être relativement faciles à distinguer et des approximations obtenues. À la limite, une analyse de discrimination (Cooley et Lohnes 1971) peut servir à classer les cas douteux. Cependant, les indices employés par RBB pour mesurer statut et qualification professionnelle ne permettant pas cette analyse.

\section{LA MULTICOLLINÉARITÉ}

Plus prosaiquement, et contrairement à l'opinion éclairée de RBB, le coefficient négatif entre PPS1 et SSE35 peut être causé par la multicollinéarité (voir Gordon, 1968 pour un exposé de ce problème). Des tests existent pour vérifier si la multicollinéarité est statistiquement significative dans une matrice de corrélation (Berk, 1977; Frane, 1977; Rockwell, 1975). Ces tests sont extrêmement sensibles au nombre de cas échantillonnés. Ils sont plus utiles pour déterminer si la matrice de corrélation est singulière ou non que pour détecter l'existence de multicollinéarité qui affecte substantiellement la valeur des coefficients de régression, du moins lorsque l'échantillon est important. En effet, avec un échantillon de 3893 individus, la statistique de Haitowsky indique que la matrice de corrélation des variables qui prédisent le SSE35 peut être inversée $\left(S_{\mathrm{h}}\right.$ se distribue comme un $\chi^{2}$ ). Cependant, il existe certaines présomptions quant à l'existence de multicollinéarité dans l'équation de régression multiple de SSE35 sur les formations scolaires (FP et FG), la qualification professionnelle à l'entrée sur le marché du travail (PPS1), le statut professionnel à cette époque (SSE1) et la qualification professionnelle à 35 ans (PPS35). Les coefficients de cheminement de causalité qui s'appliquent à SSE35 ne sont rien d'autres que des coefficients de la régression multiple de SSE35 sur les variables qui le prédisent. Si $C^{\mathrm{tj}}$ est l'inverse de la matrice de corrélation des variables indépendantes, les coefficients sont obtenus en résolvant l'équation suivante:

$$
\mathrm{C}^{\mathrm{ij}} \mathrm{R}_{\mathrm{ik}}^{\prime}=\mathrm{b}_{\mathrm{k}, \mathrm{i}}^{\prime}(\mathrm{i}=1,2, \ldots, 6)
$$

où $R_{1 k}$ est le vecteur des corrélations entre les variables indépendantes $i$ et la variable dépendante $\mathrm{k}$, et où $b_{\mathrm{k} .1}$ est le vecteur des coefficients de régression de $\mathrm{k}$ sur les $\mathrm{i}$ variables indépendantes. Par exemple, le coefficient $b_{k .3}(-.209)$ est obtenu en additionnant le produit des éléments de la troisième rangée de $\mathrm{C}^{\mathrm{j}}$ et des éléments de $\mathrm{R}_{\mathrm{ik}}$.

Un des éléments qui contribue le plus au signe négatif de $b_{k .3}$ est la valeur -.817 obtenue depuis le produit de la corrélation simple extrêmement forte du SEEl avec le SEE35 et de la valeur $C^{34}=-1.177$. Cette dernière valeur est utilisée dans le calcul de la corrélation partielle entre la qualification professionnelle à l'entrée sur le marché du travail (PPS1) et le statut socio-économique à la même époque (SSE1), cette valeur est: $r_{34.1256}=.471$, le coefficient de corrélation partielle le plus important parmi les variables indépendantes. Le coefficient de corrélation simple entre ces deux variables prend la valeur de .704. Lorsqu'il y a indépendance entre les variables indépendantes, la valeur des éléments de $C^{\jmath \jmath}$ hors diagonaux est nulle. Ces éléments, dans le cas où il y a corrélation simple entre variables indépendantes, sont comme des pondérations qui «corrigent» les effets corrélatifs. Au fur et à mesure que croissent des corrélations, augmente aussi la valeur des éléments hors diagonaux de $\mathrm{C}^{\mathrm{ij}}$. Ici, l'importance de la corrélation entre PPS1 et SSE1 contribue à la valeur négative -1.177 qui a un poids important dans la production d'un coefficient de régression négatif entre PPS1 et SSE35. Il s'agit là précisément d'un effet typique signifiant la présence de multicollinéarité. 
Par ailleurs, le coefficient de corrélation multiple entre SSE1 et l'ensemble des autres variables indépendantes est de .800 , encore une fois, le coefficient de corrélation multiple le plus important parmi ceux que l'on peut obtenir des variables indépendantes. Il faut cependant remarquer qu'aucun des coefficients n'est inférieur à .650 sauf celui du REV1 qui n'est que de .288 . Ce qui suggère soit une très importante unité conceptuelle entre les éléments de formation, de qualification professionnelle et de prestige de l'occupation, soit certaines difficultés propres aux instruments de mesure employés par RBB. Une analyse des composantes principales de la matrice de corrélation des variables indépendantes FG, FP, PPS1, SSE1, REV1 et SSE35 associe l'ensemble de ces variables dans un seul facteur sauf REV1 qui se constitue en facteur isolé. Ces deux facteurs comptabilisent $72 \%$ de la variance de la matrice de corrélation. Cette analyse ne se veut qu'une illustration des identités qui existent soit entre les concepts, soit entre les instruments de mesure utilisés. Nous ne suggérons aucunement le remplacement des six variables indépendantes par les deux facteurs dans une étude de la variation des SSE35.

\section{CONCLUSION}

Après avoir consacré deux des cinq pages de leur article qui explicitent les cheminements de causalité au thème de l'effet négatif de PPS1 sur SSE35, RBB abandonnent ce thème dans leur conclusion. Pourtant, une interprétation substantielle de cet effet aurait permis de les qualifier quelque peu.

Quatre énoncés résument les résultats de l'analyse. Les deux premiers, qui nous concernent, sont fondés uniquement sur les corrélations entre variables plutôt que sur les cheminements de causalité: 1) la carrière préserve l'ordre créé par l'école, 2) elle est un système d'imposition de l'ordre créé par l'école, 3) l'association entre qualification et statut d'emploi est invariante et, 4) la carrière est un système où les rapports entre les qualifications d'une part et le statut d'emploi et le revenu d'autre part s'accroissent. Dans la mesure où un groupe de travailleurs parmi les moins qualifiès, réussissent à atteindre un statut d'emploi plus élevé que la moyenne, la détermination mutuelle de l'école et de la carrière n'apparaît plus si absolue. Il y a donc espoir pour les non qualifiés puisque, qui ne s'instruit ou ne se qualifie pas, peut s'enrichir quand même. Ce qui, pour la C.E.C.M. et le Conseil du patronat, qui sont cités au début et à la fin de l'article, peut paraître comme une bonne nouvelle malgré les commentaires d'une autre nature de la part des auteurs.

]1 faut retenir de nos critiques qu'il y a deux façons d'interpréter le signe négatif du coefficient de régression multiple, ou coefficient de cheminement de causalité entre PPS1 et SSE35: 1) une interprétation substantielle qui requiert de nouvelles données de base analysées à l'aide d'un modèle d'analyse des résidus conforme à l'hypothèse des délais et des vacances d'emplois tel que proposé plus haut, ou 2) une interprétation plus statistique: il s'agit là d'un effet de multicollinéarité, le coefficient étant biaisé et instable. En regard de la lecture de l'inverse de la matrice de corrélation, la référence des auteurs à la reproduction de ce signe négatif lorsque l'échantillon est divisé en cohortes d'âge différent n'est pas convaincante $(46 \text {, note } 6)^{3}$. Si l'examen des nouvelles données ne permet pas de soutenir l'existence d'un modèle conforme à l'hypothèse des délais, quoique l'effet négatif se reproduise, l'utilisation de la régression par crête (ridge regression, Fenessey et D'Amico, 1980; voir aussi Gunst et Mason, 1977), peut être justifiée. Quoique les coefficients seront biaisés, ils pourront atteindre une certaine stabilité, l'effet négatif de PPS1 sur SSE35 disparaîtra probablement et surtout, les différences conceptuelles importantes entre les dimensions de l'oscupation seront préservées. Mais, en l'état actuel des choses, l'analyse de la matrice de corrélation du tableau 1 par RBB, ne permet pas de soutenir leur hypothèse des délais.

\section{BIBLIOGRAPHIE}

BERK, K.E., (1977), «Tolerance and Condition in Regression Computation», Journal of the American Statistical Association, 72: 863-866

BLAU, P.M., et O.D. DUNCAN (1967), The American Occupational Structure, New York, Wiley.

BLISHEN, B.R. et H.D. McROBERTS (1976), «A Revised Socio-economic Index for Occupations», Canadian Review of Sociology and Anthropology, 13

3. L'effet de multicollinéarité est surtout relié à la valeur de $\mathbf{C}^{34}$, soit l'inverse de la matrice de corrélation aux variables PPS1 et SSEI. Que cette valeur soit stable pour plusieurs cohortes n'est guère surprenant puisque la qualification (PPS1) est mesurée depuis les emplois. Le rapport d'une qualification à un emploi spécifique étant constant quelque soit la cohorte 
CARLOS, S., (1970), «Les cheminements de causalité», Sociologie et sociétés, 2: 189-202.

COOLEY, W.W. et P.R. LOHNES (1971), Multivariate Data Analysis, New York, Wiley.

DUNCAN, O.D., (1975), Introduction to Structural Equation Models, New York, Academic Press.

DRAPER, N. et H. SMITH, (1966), Applied Regression Analysis, New York, Wiley.

FENESSEY, J, et R. D'AMICO (1980), «Collinearity, Ridge Regression, and Investigator in Judgment», Sociological Methods and Research, 8: 309-340.

FRANE, J.W., (1977), «A Note on Checking Tolerance in Matrix Inversion and Regression», Technometrics, $19: 513-514$. GORDON, R.A. (1968), «Issues in Multiple Regression», American Journal of Sociology, 73: 592-616.

GUNST, R.F. et R.L. MASON (1977), «Advantages of Examining Multicollinearities in Regression Analysis», Biometrics, 33: 249-260.

RENAUD, J., P. BERNARD et M. BERTHIAUME (1980), «Éducation qualification professionnelle et carrière au Québec», Sociologie et sociétés, 12: 1, 23-52.

ROCKWELL, R.C., (1975), «Assessment of Multicollinearity: The Haitowsky Test of the Determinant», Sociological Methods and Research, 3: 308-320. 